\title{
Louisa Burns Memorial Lecture: Reform through research
}

\author{
ANTHONY G. CHILA, D.O., FAAO, OUCOM
}

Athens, Ohio

In keeping with the specifications of the Louisa Burns Memorial Lecture, my comments will reflect my association with osteopathic research as well as my observations as a clinician offered to the research community of the osteopathic profession. In so doing, the process of osteopathic education and research as I have experienced it will be reviewed.

Acquiring an osteopathic medical education during the years 1961-1965 was a tenuous process at best. In the early months of 1962, formal amalgamation of the allopathic and osteopathic professions occurred in the state of California. This event caused the loss of one of the then six colleges of osteopthic medicine, many hospitals, and approximately 2000 osteopathic physicians. Licensure for future osteopathic physicians was eliminated in the process. As one of the osteopathic profession's darkest hours, the completion of this merger simply represented the culmination of years of exploratory interchanges between representatives of both professions. It should be noted that the California experience was regarded as a model for subsequent, progressive amalgamation of the allopathic and osteopathc professions throughout the United States. In the state of Washington during the years 1962-1963, dissident osteopathic physicians gained support from the State Medical Society which led to the founding of a "college" to award M.D. degrees to "qualified" D.O.s practicing in Washington. In the state of New York in 1964, legal action to secure the privilege of having licensure designation as D.O. and M.D. was sought by an osteopathic physician. By 1965 , the professional and political turmoil of those years made it a source of wonder that a graduate actually received a D.O. degree-it was certainly a surprise to me.

The attitude of a student and graduate of that period was unavoidably influenced by the uncertainties arising from these excursions into oblivion. It was often difficult to remain convinced about the wisdom of having chosen a minority profession of the healing arts which argued for separatism because of its distinctive philosophy and contributions to health care delivery, while being apparently unable (or unwilling) to define and pursue this distinctiveness. This enigma called osteopathic medicine was comprehensively portrayed by sociologist Norman Gevitz. ${ }^{1}$

The osteopathic profession's losses of identity as encountered during the $1960 \mathrm{~s}$ were largely recovered by 1974 . In that year, which interestingly coincided with the centennial of Andrew Taylor Still's formal articulation of his premises of osteopathy, unlimited licensure for osteopathic physicians became a reality in 50 states and the District of Columbia. Even this accomplishment, however, did not assure sustained commitment to the development of Still's philosophy of osteopathy.

Since commencing the study of osteopthic medicine in 1961, I have been privileged to experience the private practice arena as well as to participate in the educational environment of this profession. In both, I have been involved in predoctoral and postdoctoral education. In the latter, I have experienced administrative responsibilities and involvement in the development of research activities. The remainder of this discussion will be devoted to aspects of these experiences which have led me to believe that reform of osteopathic medicine's role in its contribution to health care delivery can come through greater acceptance of the obligation to provide evidence of research committed to its premises.

\section{Missed opportunities}

During the years that I was engaged in private practice (1966-1977), two situations which could have markedly influenced the sustained course of osteopathic research were not appropriately utilized by the profession. Inasmuch as elements of these situations remain viable today, perhaps the opportunities are still not lost.

The golden age of osteopathic research In the present era of affluence of osteopathic medicine, 15 colleges enjoy increased levels of state and federal support, and several have university affiliations. It is interesting to note that despite this growth and success, research programs by and large are not clearly directed by considerations of osteopathic philosophy. The participation of clinicians in basic science studies is relatively sparse, and efforts to train individuals as clinician-scientists (D.O.-Ph.D.) have not yet been overly fruitful.

The modern era of osteopathic research was initiated through the professional efforts of J. Stedman Denslow, D.O., (1906-1982). An osteopathic clinician, Doctor Denslow devoted his career to the study and investigation of the basic scientific premises of his profession. He led the first group of osteopathic physicians who sought to develop guidelines and secure support for research performed under os- teopathic auspices. Doctor Denslow coauthored the first paper to be published by a Doctor of Osteopathic Medicine in a refereed journal devoted to research. ${ }^{2}$

The larger picture of Doctor Denslow's contribution to the development of osteopathic research is set in the character of research performed at the Kirksville College of Osteopathic Medicine during the years $1938-1978$. The highly productive studies accomplished by clinicians and basic scientists interfacing with each other helped to shape a series of publications dedicated to the clarification of osteopathic viewpoints. The names of Korr, Krems, Wright, Thomas, Hix, Chace, Cole, and others attest to the sustained motivation of this period of time. Outcomes included studies on electromyography, the sympathetic nervous system, reflexes, axonal transport, and the trophic functions of nerves. When seen in the context of osteopathic philsophy and practice, the impact is overwhelming. The American Academy of Osteopathy made available to its members The Collected Papers of Irvin M. Korr. ${ }^{3}$ In so doing, a tribute to an outstanding basic scientist became also a compilation of the group's accomplishments. This volume should be considered a major resource for anyone seeking to understand osteopathic orientation in research.

The legacy of the Kirksville group lies in its utilization of clinicians and basic scientists individually contributing expertise. The common goal was the commitment to the study of osteopathic premises. The group's studies heavily emphasized "the facilitated segment"; the broader meaning of somatic dysfunction was in the early stages of development and acceptance when the group ceased to function. In retrospect, it is sad that the productive effort of this group was not complemented by clinical research studies dealing with active disease processes.

\section{The hospital service of osteopathic} medicine

Beginning in 1973, Edward G. Stiles, D.O., in cooperation with the Board of Trustees of Waterville (Maine) Osteopathic Hospital, sought to implement the profession's first hospital-based service of osteopathic medicine. ${ }^{4}$ Doctor Stiles' experiences speak to the willingness of a Board of Trustees to define and initiate steps to assist an institution in the realization of its osteopathic potential. His experiences also speak to the lack of appreciation of manipulative treatment in total health care management in the various clinical disciplines. 
The Waterville experience began with the appointment of Doctor Stiles as the hospital's Director of Osteopathic Medicine. This effectively defined a service which would prevail throughout the hospital. The director of this service was salaried and employed by the Board of Trustees. This indicated to the attending staff and public the Board's dedication to the utilization of osteopathic philosophy in health care management. The director functioned as a consultant to the attending staff for both inpatients and outpatients. This permitted the use of educational methods to increase the attending staff's utilization of palpatory diagnosis and osteopathic manipulative treatment.

Realization of the importance of these considerations led to the appearance of many services of osteopathic medicine in hospitals throughout the profession. Failure to adhere to the significance of these considerations ultimately diluted the impact of the Waterville experience. The passage of time has shown that changing social demands and external pressures for cost containment are probably responsible for causing a shift from hospital support to ambulatory service. The weakening of the educational strategy of such a service ultimately contributed to the continuing abrogation of responsibility to provide a distinctive quality of care in osteopathic community hospitals. ${ }^{5}$

The hospitals of the osteopathic profession did not successfully pursue the larger implications of the Waterville experience. If this had been done, it is safe to say that a foundation could have been prepared for multicenter clinical research studies dealing with osteopathic management of active disease process.

\section{Contemporary osteopathic education}

Since 1977 , I have been professionally in volved in osteopathic medical education. My first assignment was with Michigan State University College of Osteopathic Medicine. I was recruited from private practice to establish the service of osteopthic concepts and methods at Lansing (Michigan) General Hospital. During this affiliation, I completed a palpatory evaluation of somatic findings in $27 \mathrm{pa}-$ tients who had recently been diagnosed as having acute myocardial infarction. The study was not published, but findings were shared with members of the MSUCOM clinical faculty. This experience significantly stimulated my interest in the implication of change over time as a risk factor associated with somatic reflectance potentially involved in active disease processes. The opportunity to affiliate with the newly developing Ohio University College of Osteopathic Medicine in 1978 provided me with the opportunity to develop clinical research studies as part of the educational process of osteopathic medical students.

Multicenter clinical research studies

The state of Ohio has, for many years, ranked third in the osteopathic profession in terms of organizational strength. This refers to the total number of practicing osteopathic physicians, the total number of teaching hospitals, their bed capacity, and variety of postdoctoral programs, and the presence of a college of osteopathic medicine. From its inception, the Ohio College perceived its educational role to be interrelated with the state's professional resources. Over time, it has become increasingly obvious that great mutual benefit exists for the quality of education through this interrelatedness. The College's participation in the academic tradition of Ohio University is a major consideration in this development.

It has been possible to utilize this professional and educational environment to develop a strategy for the implementation of multicenter clinical research studies. ${ }^{6}$ The objective of this strategy is to facilitate and enhance the utilization of palpatory diagnosis and osteopathic manipulative treatment in the educational process. The focus of this strategy is to establish an educational interface among students, interns, residents, and attending physicians in osteopathic teaching hospitals. In my experience with developing this strategy since 1978 , I wish to acknowledge the following individuals for their administrative and collegial support of my efforts: Dean Frank W. Myers, OU-COM; Norman J. Larson, D.O.; Albert F. Kelso, Ph.D., and Michael M. Patterson, Ph.D.

\section{Organizational format}

In my capacity as Chief of Clinical Research at OU-COM, I serve as the principal investigator for our regional site clinical research studies. The support of hospital administration, clinical staff, and nursing staff has been enlisted through an ongoing educational process. Selected clinicians in various specialty areas have been designated as associate investigators for the various studies. Voluntary participation is encouraged for all students at the various regional sites. Those who actively participate are designated as student investigators and are given specific training in palpatory diagnosis and/or osteopathic manipulative treatment appropriate to the particular study. Requisite acknowlegement of human subjects' safety is provided for in any study undertaken. The educational emphasis of these studies is compatible with goals expressed in basic documents of the American Osteopathic Association in regard to postdoctoral training.

\section{Outcomes}

Pneumonia, 1978-1983. The choice of an appropriate disease model is crucial to the successful design and implementation of a clinical research study. Our initial effort dealt with pneumonia. This choice was made because of the widespread belief of clinicians that efficacy of osteopathic manipulative treatment could be more readily demonstrated in disorders of the respiratory system. This choice was also made because of the generally accepted historical success of osteopathic physicians utilizing osteopathic manipulative treatment in the management of influenza and pneumonia prior to the development of antimicrobial agents.

The protocol which was developed dealt with the patient hospitalized with a diag nosis of pneumonia. Criteria were established for the selection and entry into the study of pediatric patients ( 6 to 16 years of age) and adult patients (16 years of age and older). Exclusion criteria were defined. Random selection was determined for patient designation as a control or ex perimental subject. Stages of illness were defined. Osteopathic structural evaluation was defined for both control and experimental patients. Osteopathic manipualtive treatment was defined for experimental patients and administered according to the stage of the illness. ${ }^{7}$

Despite this depth of preparation, problems were encountered as follows: limited participation by clinicians willing to serve as associate investigators, inconsistent criteria for diagnosis of pneumonia as employed by practicing physicians; seasonal variation in the clinical appearance of pneumonia, rendering difficulty in securing an adequate patient population. For these reasons the study was administratively discontinued in 1983.

Acute myocardial infarction, 1984-1987. Many of the difficulties encountered in the pneumonia study were eliminated by the choice of acute myocardial infarction as a subject for study. Diagnostic criteria are more clearly established and utilized by practicing physicians; a patient population is more readily secured because of stringent criteria for management, intervention and rehabilitation of patients; this disease entity is not influenced by seasonal factors. The most noteworthy impact of this choice of subject was the willingness of physicians to serve as associate investigators for the study. A specialist in internal medicine from each of the College's affiliated teaching hospitals is actively involved in the development of this study. The study to date has been limited to a single regional palpatory examination performed as a patient was released from the Intensive Care Unit and brought to "step-down care." Findings referable to cervical column segmental dysfunction have been compared with a previous study. ${ }^{8}$ Further study of vertebral and paravertebral reactions of the thoracic column as well as reactive hyperemia is pending.

Educational response. Despite the various difficulties encountered in the selection of 'a disease model for study, and the design of the study, interest is sustained. The experiences at OU-COM since 1978 have established a model for the improvement of osteopathic education beyond the basic didactic years. Support exists for the continuation of this model. 
In terms of student participation, the following documentation is established for the OU-COM classes of 1981-1987 inclusive: 1981,14 percent; 1982,16 percent; 1983, 31 percent; 1984,15 percent; 1985 , 25 percent; 1986,14 percent; and 1987, 14 percent. I am indebted to those students, now graduates of OU-COM, who gave of their time and effort in the support of these studies. I hope that future years will see increasing numbers of student participants.

\section{Somatic dysfunction and change over} time

The osteopathic profession has, for many years, wandered from the path of its basic philosophy. The use of manipulations is central to and not synonymous with the philosophy of osteopathy. This implies the use of palpatory diagnosis in any area of osteopathic medical practice. It also implies consideration of the use of osteopathic manipulative treatment in the total management of a patient's disorders. This view speaks to evaluation and not only examination; principle and not only technique or etiology. Most important is the consideration of disease as effect. In order for this profession to remain viable in its role in health care delivery, it is mandatory that clinicians reflect more clearly a practice based on this philosophy. In order for this profession to establish an assertive role in its contribution to research, it is necessary that its researchers be willing to consider implications of this philosophy in research design. Palpatory diagnosis applied to considerations of impaired or altered function documented, observed, and treated over time, should be the basis of future research activity for this profession. The 1986 request for proposals of the American Osteopathic Association Bureau of Research reasonably embodies such concerns. ${ }^{9}$

The American Osteopathic Bureau of Research ad hoc Committee on Recording Musculoskeletal Findings

I have been involved with the activities of the Bureau of Research since 1980, first as a member of the Bureau and currently as a member of its Committee on Research Grants. In 1985, at the charge of the $\mathrm{Bu}$ reau of Research, I was asked to chair an ad hoc Committee on Recording Musculoskeletal Findings. This committee met at Lansing, Michigan, on November $2-3,1985$, and prepared a report which was submitted to the Bureau of Research for its meeting in March of 1986.

The Committee consensus was that it should be responsible for developing guidelines to be used by individual investigators in the development of protocols. It was also felt by the Committee that osteopathically oriented studies need to elaborate upon generally accepted criteria for research studies.

In reaching its conclusions, the Committee first considered traditional components of clinical research studies:

1. Conventional clinical research stud- ies are generally described as being: (a) descriptive (having no intervention); or (b) experimental (having an intervention). Guidelines for such studies may be cited that measure an effect by requiring: (a) criteria for inclusion/exclusion; (b) criteria for the dependable variable(s) of the study; (c) criteria for the independent variable(s) of the study; (d) application of a procedure/treatment; and (e) measurement of an effect.

2. From a different philosophic view, Doctors of Osteopathic Medicine have dealt with studies which have attempted to: (a) describe the musculoskeletal examination, test procedures, and positive findings; (b) observe the effect(s) of a manipulative procedure applied to an individual; and (c) establish a clinical diagnosis and observe the effect(s) of administering osteopathic manipulative treatment.

3 . It becomes readily apparent, then, that differing philosophies should provide differing criteria for clinical research studies done under the auspices of a particular philosophy.

4. In the case of osteopathic clinical research studies, then, criteria such as the following are more appropriate to the intent of the philosophy underlying the proposed study: (a) criteria for inclusion exclusion of somatic dysfunction; (b) criteria for the dependent variable(s) of the study (somatic dysfunction, other tissues, and functions); (c) criteria for the independent variable(s) of the study (somatic dysfunction being present); (d) application of a procedure/treatment (somatic dysfunction being absent); and (e) measurement of an effect(s) on somatic dysfunction, other organs or tissues, body structure or function.

5 . Such an elaboration of the conventional requiremnts for clinical research studies helps to establish a framework suitable to be referred to as osteopathic clinical research.

The report of the Committee was as follows:

\section{A. Summary statement}

The guidelines for investigators offered herein represent the Committee's view of an appropriate procedure for determining the components of and recording the use of palpatory diagnosis and osteopathic manipulative treatment. It is the Committee's consensus that these guidelines should reflect the differentiation of osteopathic philosophy which helps to elaborate the criteria generally utilized by investigators in conventional clinical research studies. It is also the Committee's consensus that these guidelines should help to make a distinction between a manipulative procedure and osteopathic manipulative treatment as defined in clinical research studies. It is finally the Committee's consensus that these guidelines must be included in clinical research studies conducted under osteopathic auspices.

\section{B. Determining the components of}

somatic dysfunction

1. Musculoskeletal examination

(a) Description of tests utilized for determining the presence or absence of somatic dysfunction

(b) Criteria for positive findings

(c) Description of findings relating to the assessment of somatic dysfunction

2. Preliminary musculoskeletal diagnosis

3. Intervention (osteopathic manipulative treatment)

(a) Based on test findings

(b) Objective of treatment

(c) Description of treatment procedure(s) used

4. Reassessment

(a) Reevaluation of findings, utilizing original tests for somatic dysfunction

C. Documenting the effects of osteopathic manipulative treatment

1. Local musculoskeletal effects

2. Remote musculoskeletal effects

3. Other effects
a. Behavioral
b. Visceral
c. Communicating

I wish to express appreciation to the following individuals for their participation in the deliberations of the Committee: Gilbert E. D'Alonzo, D.O., ViceChairman; Myron C. Beal, D.O.; John P. Goodridge, D.O.; William L. Johnston, D.O.; Robert E. Kappler, D.O.; Albert F. Kelso, Ph.D.; and Michael M. Patterson, Ph.D.

\section{Osteopathic purpose}

The educational process of the osteopathic profession has been the focus of this discussion. Particular emphasis has been placed on selected events which have occurred during the past 25 years. During this period of time, and longer, the end product of the process has been identified as a unique and distinct provider of health care. Justification for a separate professional existence has long been predicated on this concept. In itself, this attitude does not adequately consider the purpose of the osteopathic profession. ${ }^{10}$ This attitude has further tended to downplay the appreciation of a fundamental need in the clinical arena. In order to revitalize its appreciation of its purpose, the osteopathic profession must establish better communication between basic research activity and the use of palpatory diagnosis and osteopathic manipulative treatment in clinical practice. Basic research is the foundation for understanding the premises of osteopathic philosophy. In order to avoid a serious intellectual vacuum, widespread and large-scale integrated activity involving basic researchers and osteopathic clinicians is sorely needed. The combined meetings of this conference and the American Academy of Osteopathy convocation programs in 1984 and 1985 were 
an attempt to stimulate such activity.

As considered by Polanyi, 11 passionate preoccupation with a problem alone can elicit discovery. The announcement is often followed by struggles against doubt. The struggle becomes transformed into a craving to convince, which is a process of verification. Andrew Taylor Still's preoccupation was well described by Kimberly ${ }^{2}$ as the welding of the principles of body unity, the healing power of nature, and structure-function interrelationships with the concept that deranged soma will result in altered physiology and/or chemistry of related tissues. The result was a philosophy which Still named osteopathy and which he intended to guide the medical world in improvement upon its system. It would be well for us to incite ourselves to passionate preoccupation with the implications of Still's philosophy as we consider research strategies and undertakings. It behooves us to contrib- ute to the fulfillment of the challenge of this philosophy. In so doing, we would be more likely to meet unfulfilled social needs. This would provide evidence of osteopathic medicine's commitment to its original purpose of medical reformation as envisioned by Andrew Taylor Still.

1. Gevitz, N.: The D.O.'s: Osteopathic medicine in America. John Hopkins University Press, Baltimore, 1982

2. Denslow, J.S., and Clough, G.H.: Reflex activity in the spinal extensors. J Neurophysio 4:430-437, Sept 41

3. Peterson, B., ed.: The collected papers of Irvin M. Korr. American Academy of Osteopathy, Colorado Springs, Colorado, 1979

4. Stiles, E.G.: Osteopathic medicine. Distinction or extinction? Scott Memorial Lectures American Academy of Osteopathy, Newark. Ohio, 1985, pp. 41-5

5. Chila, A.G.: Quality of care. An assessment of the contributions of osteopathic medicine.
The D.O. pp. 99-108. May 78

6. Chila, A.G.: Multicenter clinical research studies. Organizational strategy. JAOA 86:674, Oct 86

7. Chila, A.G., Patterson, M.M., and Burns, D.G.: Pneumonia. A clinical research protocol JAOA 80:751/97-2/8, Jul 81

8. Chila, A.G., et al:: Somatic dysfunction in acute myocardial infarction. The student investigator. Presented at AOA/NOF Research Conference, Clearwater Beach, Florida, 1987

9. American Osteopathic Association, Bureau of Research ad hoc Committee on Recording Musculoskeletal Findings, 1985: Report submitted to the Bureau of Research, March 1986 10. Korr, I.M.: Osteopathic medicine's forgotten purpose. JAOA 87:100-1, Feb 87

11. Polanyi, M.: Personal Knowledge. The University of Chicago Press, Chicago, (1958; corrected edition 1962), p. 171

Dr. Chila, is professor and chairman, Department of Family Medicine and chief of clinical research, Ohio University College of Os. teopathic Medicine, Athens, Ohio 45701. 